\title{
Filter design for failure detection and isolation in the presence of modeling errors and
} disturbances

Niemann, Hans Henrik; Stoustrup, Jakob

Published in:

Proceedings of the 35th IEEE Conference on Decision and Control

Link to article, DOI:

10.1109/CDC.1996.572630

Publication date:

1996

Document Version

Publisher's PDF, also known as Version of record

Link back to DTU Orbit

Citation (APA):

Niemann, H. H., \& Stoustrup, J. (1996). Filter design for failure detection and isolation in the presence of modeling errors and disturbances. In Proceedings of the 35th IEEE Conference on Decision and Control (Vol. Volume 2, pp. 1155-1160). IEEE. https://doi.org/10.1109/CDC.1996.572630

\section{General rights}

Copyright and moral rights for the publications made accessible in the public portal are retained by the authors and/or other copyright owners and it is a condition of accessing publications that users recognise and abide by the legal requirements associated with these rights.

- Users may download and print one copy of any publication from the public portal for the purpose of private study or research.

- You may not further distribute the material or use it for any profit-making activity or commercial gain

- You may freely distribute the URL identifying the publication in the public portal 


\title{
Filter Design for Failure Detection and Isolation in the Presence of Modeling Errors and Disturbances*
}

\author{
H.H. Niemann ${ }^{\dagger} \quad$ J. Stoustrup ${ }^{\ddagger}$
}

\begin{abstract}
The design problem of filters for robust Failure Detection and Isolation, (FDI) is addressed in this paper. The failure detection problem will be considered with respect to both modeling errors and disturbances. Both an approach based on failure detection observers as well as an approach based on a standard setup optimization is presented in this paper.
\end{abstract}

\section{Introduction}

The main problem in failure detections for dynamic control systems is to distinguish failures from other disturbances, facing model uncertainties in the system. In the past $10-15$ years, a lot of papers has been considering the failure detection problem. Good survey papers can be found in [11] and in [4]. Further, the book by Patton et al. [10], also gives a good introduction to the area of failure detection.

In the past years, the robustness aspect has also been introduced in relation with failure detection as e.g. in [12], [1], [13], [3] and in [9] to mention a few papers.

The FDI methods described in this paper are related to other methods derived in this area which are either based on observer techniques or on an optimization approach, like $\mathcal{H}_{\infty}$ or $\mathcal{L}_{1}$ or combinations of these. These two types are not the only types of methods for failure detections and isolations. Another class of methods is based on statistical approaches, see [7].

If we consider filters for FDI based on optimizations design, a lot of different methods has been derived. A general observer based approach in [5] has been

\footnotetext{
${ }^{\dagger}$ Department of Automation, Technical University of Denmark, Building 326, DK-2800 Lyngby, Denmark.

E-mail: hhneiau.dtu.dk

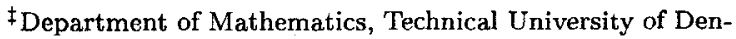
mark, Building 303, DK-2800 Lyngby, Denmark.

WWW: http: //wru .mat .dtu .dk/IEEE/js . html

E-mail: jakob@mat.dtu.dk

* This work is supported by the Danish Technical Research Council under grant. no. 9500765
}

applied in connection with an $\mathcal{H}_{\infty}$ optimization. A result of these methods can be found in [6], where the benchmark problem from [2] has been considered. A direct formulation of the FDI problem as a robust $\mathcal{H}_{\infty}$ filter design problem has been given in [3]. This approach results in solving a standard Riccati equation for a standard $\mathcal{H}_{\infty}$ filter problem. Another $\mathcal{H}_{\infty}$ approach to the FDI problem can be found in [12]. An approach based on $\mathcal{H}_{\infty}$ optimization is given in [9], where the robust FDI design is combined with statistical methods for the detection of failure signals. The result given in the paper is very convincing.

The reason for using $\mathcal{H}_{\infty}$ optimization in connection with the FDI problem is due to the robustness aspect with respect to model uncertainties. However, from a FDI point of view, it is more natural to use an $\mathcal{L}_{1}$ optimization of the FDI filter. By using an $\mathcal{L}_{1}$ optimization of the FDI filter, we will obtain that the magnitude of the residual signal with respect to disturbances and model uncertainties are bounded in magnitude. This will make the detection of failure signals more readily comparable to the case where an $\mathcal{H}_{\infty}$ optimization has been applied. In this case, the residual signal is bounded in the power semi-norm. An $\mathcal{L}_{1}$ optimization for integrated control and FDI has been applied in [1].

The rest of this paper is organized as follows. First, in Section 2 we describe an observer based solution. This setup is quite intuitive. Based on this setup, we formulate the robust failure detection problem in a standard problem framework in Section 3, and we provide several approaches to its solution based on optimization methods. Section 4 summarizes the results.

\section{An Observer Based FDI Ap- proach}

We shall follow the observer based approach derived by Patton et al. described in e.g. [10], [11] or in [8]. Together with the observer, an output weight matrix is included as an additional design parameter. In this section we shall demonstrate how to modify 
the observer structure in order to allow for systematic design of the weight matrix and the observer simultaneously by formulating it as a standard robust design problem. In contrast, using a standard setup, the design of the observer and the weight matrix would be coupled.

Consider the following system $G$ given by:

$$
\begin{aligned}
& \dot{x}=A x+B_{1} w+B_{2} d+B_{3} f \\
& y=C x+D_{1} w+D_{2} d+D_{3} f
\end{aligned}
$$

(We shall throughout the paper assume 'compatible' dimensions of vectors and matrices to avoid tedious listings of dimensions.)

Now, let's use the failure detection approach described in e.g. [11] or in [8], where an observer together with a weight matrix are applied for the failure detection. Let the full order observer be given by:

$$
\dot{\hat{x}}=A \hat{x}+K(y-C \hat{x})
$$

where $K$ is the observer gain and $\hat{x}$ is the state estimate. The output error $e_{y}$ is given by

$$
e_{y}=y-C \hat{x}
$$

By using the observer on the system in (1), we get the following equation for the output error $e_{y}$

$$
\begin{aligned}
e_{y}= & C(s I-A-K C)^{-1}\left(B_{1}+K D_{1}\right) w \\
& +C(s I-A-K C)^{-1}\left(B_{2}+K D_{2}\right) d \\
& +C(s I-A-K C)^{-1}\left(B_{3}+K D_{3}\right) f \\
& +D_{1} w+D_{2} d+D_{3} f \\
= & G_{e w}(K, s) w+G_{e d}(K, s) d+G_{e f}(K, s) f
\end{aligned}
$$

To realize a failure detection and isolation scheme, we will premultiply the output error $e_{y}$ by a prefilter $H(s)$, to generate the residual:

$$
\begin{aligned}
r(s)= & H(s) G_{e w}(K, s) w+H(s) G_{e d}(K, s) d \\
& +H(s) G_{e f}(K, s) f
\end{aligned}
$$

The design parameters are then the observer gain $K$ and the filter matrix $H$. The design of these matrix gains are done separately in the design method applied in [11], [8]. The observer gain is designed by an Eigenstructure Assignment method and the following design of $H$ is more ad hoc. However, here we want to derive a combined design method for the observer gain and the prefilter by formulating the design problem in a standard setup. First. let's look at the design conditions we have to the transfer matrices from $w, d$ and $f$ to $r$ in (4). It is clear, that we want to minimize the transfer matrix from the external input $w$ to $r$ and also the transfer function from $d$ to $r$. These transfer function matrices represent model uncertainties and disturbances, which we want to make the residual robust against. The transfer matrix from the failure signals $f$ to the residual $r$ must not be small, due to the fact that we want to detect the failure signals in the residual vector. Therefore, we can instead look at the transfer matrix given by $I-H G_{e f}$. By minimizing this transfer matrix, we will obtain that the residual vector gives a good estimate of the failure vector. To summarize, we have the following design conditions for the design problem:

Problem 1 Let the residual vector be given by

$r(s)=H G_{e w}(K, s) w+H G_{e d}(K, s) d+H G_{e f}(K, s) f$

where the controller parameters are $K$ and $H$. Design $K$ and $H$ such that

$$
\begin{aligned}
& H G_{e w} \approx 0 \\
& H G_{e d} \approx 0 \\
& H G_{e f} \approx I
\end{aligned}
$$

The conditions will not be possible to meet uniformly in frequency, but should be met at "appropriate frequencies". We will elaborate on this below.

After a little matrix manipulation, the transfer matrix from $w, d$ and $f$ to $r$ can be described as an LFT in the controller parameters $K$ and $H$, i.e. $\mathcal{F}_{l}(\tilde{G}(s), \tilde{K}(s))$, where $\tilde{G}(s)$ is given by

$$
\tilde{G}(s)=\left[\begin{array}{c|ccccc}
A & B_{1} & B_{2} & B_{3} & {\left[\begin{array}{ll}
I & 0
\end{array}\right]} \\
\hline 0 & 0 & 0 & -I & {\left[\begin{array}{ll}
0 & I
\end{array}\right]} \\
C & D_{1} & D_{2} & D_{3} & {\left[\begin{array}{ll}
0 & 0
\end{array}\right]}
\end{array}\right]
$$

and the controller $\tilde{K}(s)$ is given by

$$
\tilde{K}(s)=\left[\begin{array}{l}
K \\
H
\end{array}\right]
$$

In the original design setup by Patton et al. [11], the two gains $K$ and $H$ are constant gains, but this will not in general be the case now. Here we will get two dynamic controllers which will satisfy the design conditions. By just using constant gains, we will not in general get an optimal observer/filter.

The structure described here is depicted in Figure 1.

The $\Delta(s)$ block represent the robust stability condition. The $\Delta_{f}(s)$ and $\Delta_{d}(s)$ blocks represent performance specifications, i.e. specifications for the disturbance attenuation and for the performance specification for the failure signals. 

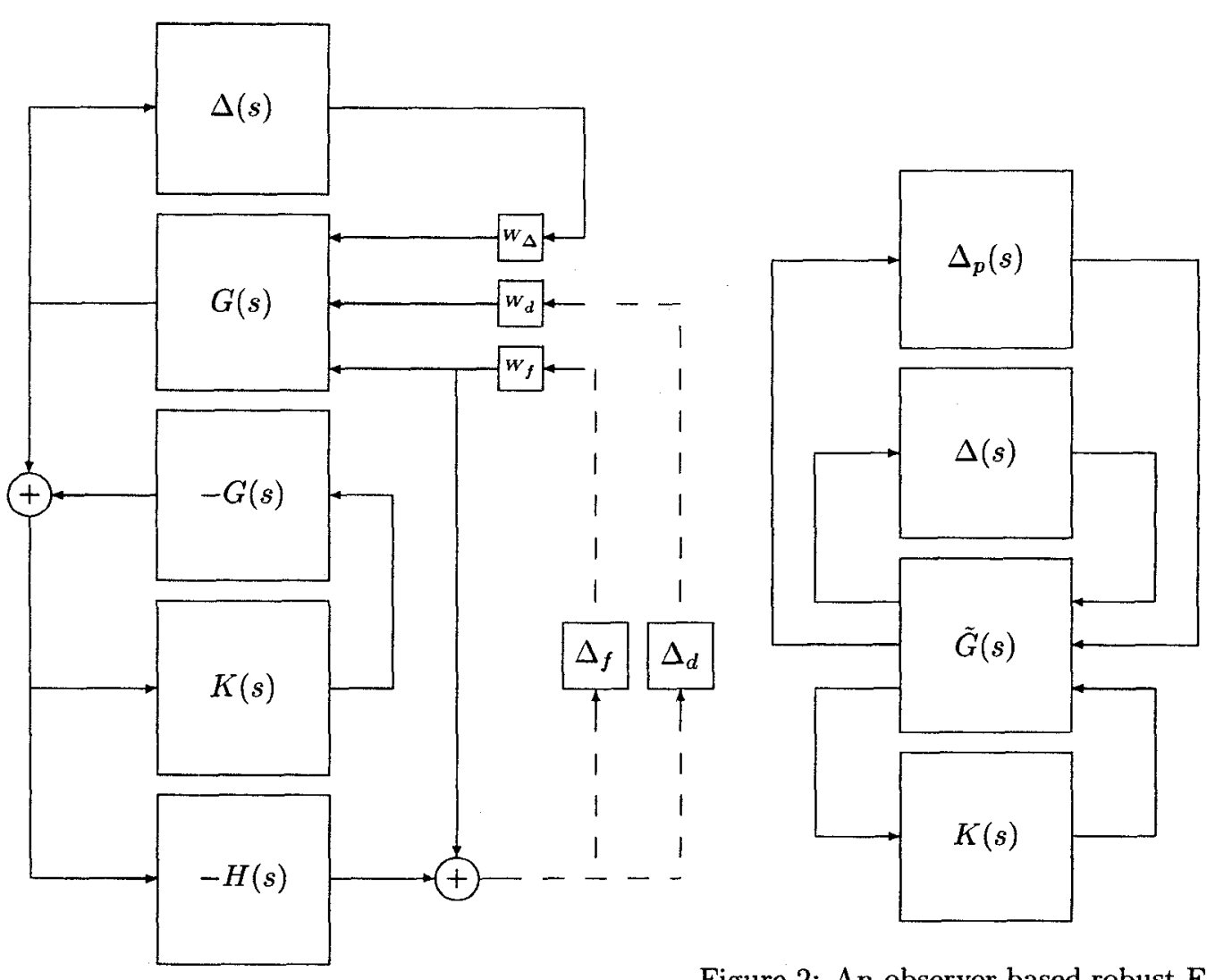

Figure 2: An observer based robust FDI structure in a $\mu$ or $\mathcal{L}_{1}$ setup

Figure 1: An observer based robust FDI structure

The design problem of an observer for failure detection has now been transformed into a standard setup as shown in Figure 1. By using this setup, the two controllers, $K$ and $H$ are designed in one step. Moreover, the design conditions for the filter $\tilde{K}$ has explicitly been formulated and applied in the setup.

Based on the design setup shown in Figure 1, it is easy to derive a setup as shown in Figure 2, where the observer design FDI problem is formulated in a more standard way.

Let's again consider the block diagram shown in Figure 1. By observing that the controller is described by the three blocks including $-G(s), K(s)$ and $-H(s)$, it is possible to make a simplification. The "controller" for the system $G(s)$ in Figure 1 is given by

$$
\begin{aligned}
u(s) & =-H(s)(I+G(s) K(s))^{-1} y(s) \\
& =F(s) y(s)
\end{aligned}
$$

It is clear from (7) that we do not necessarily wish to formulate the FDI problem as an observer and a prefilter if we afterwards are going to design the controller by an optimization method in one step. Instead, we can go directly after a filter $F(s)$ that will

satisfy our design conditions. The setup can then be described by the block diagram shown in Figure 3.

By using the equation for the filter based on an observer in (7), we can see that there is an additional freedom in the observer based approach in comparison to the filter version. With this freedom, we can e.g. see that we can obtain an arbitrary controller transfer function $F(s)$ by selecting the prefilter $H(s)$ as

$$
H(s)=-F(s)(I+G(s) K(s))
$$

with an arbitrary stabilizing observer gain $K(s)$.

With these remarks about the observer FDI structure we will conclude this section by remembering that the observer based FDI approach is very well motivated in its setup. Moreover, if a two step design approach is applied as in [11], [8], the observer setup is very useful. However, in terms of performance, an observer based structure does not offer any advantages compared to a filter based FDI solution. 


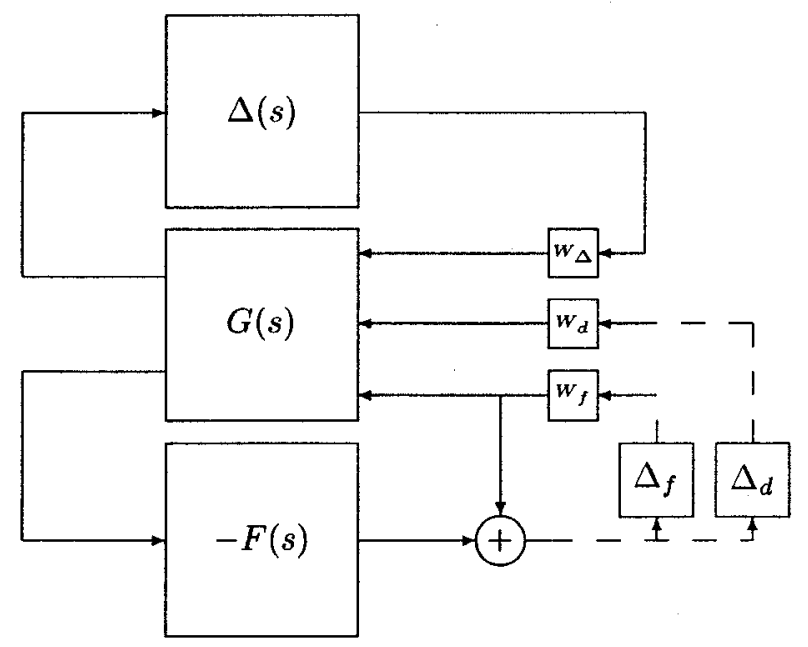

Figure 3: A filter based robust FDI structure

\section{Standard Problem Formula- tion}

The approach in this section is appealing in the sense that it is based on a very straightforward and precise problem formulation for the robust FDI problem which can then be solved in one step. Based on the setup in Figure 3, we will derive the setup directly. The structure of the controller will not, though, be intuitive in any sense as in the case where an observer is applied. We consider the following model for a (potentially) faulty uncertain dynamical system:

$$
\begin{aligned}
& \dot{x}=A x+B_{1} w+B_{2} d+B_{3} f \\
& z=C_{1} x+D_{11} w+D_{12} d+D_{13} f \\
& y=C_{2} x+D_{21} w+D_{22} d+D_{23} f
\end{aligned}
$$

$x$ is the state vector. $w$ and $z$ describe the uncertainty dynamics, i.e., the uncertainty is described as a feedback interconnection from $z$ to $w$ with a feedback of the form $W_{\Delta} \Delta,\|\Delta\|_{\infty}<1$. $W_{\Delta}$ describes the frequency dependence of the uncertainty. This setup is standard and fairly general.

$d$ is the vector of external disturbances and noises. By selecting matrices $B_{2}$ and $D_{22}$ carefully, it is possible to model both the exogenous signals that enter the dynamics as well as those that influence the measurements only.

$f$ is the failure vector, which include both sensor and actuator failures (by selection of the $B_{3}$ and $D_{23}$ matrices).

Finally, $y$ is the measurement output.

We shall now set up an optimization problem in standard form. In order to obtain useful results it is of paramount importance to introduce appropriate weightings. In addition to the uncertainty model $W_{\Delta}$ which will typically possess high pass properties, we need weightings that describe the disturbance and fault models. Hence, we assume the signal $d$ to be generated by a frequency unbiased signal $w_{2}$ passing through a filter $W_{d}$ :

$$
d=W_{d} w_{2}
$$

Typically, $W_{d}$ will be of low pass character if it describes process disturbances and of high pass character if it is a sensor noise model.

Similarly, we assume $f$ to be generated by a signal $w_{3}$ with flat frequency characteristics:

$$
f=W_{f} w_{3}
$$

Typically $W_{3}$ will have a low pass character. If only static failure signals are anticipated (as will often be the case) it is important to assign the roll off frequency for this filter much lower than the system cross over frequency.

The objective of our optimization is to generate a signal $u$ which approximates the faults $z$ as well as possible. In other words, we wish the following error:

$$
z_{2}=u-f
$$

to be as small as possible, subject to the modeling error $\Delta$, and in presence of the exogenous signals $d$.

Assuming state space models of the three weights as follows:

$$
W_{i}=\left[\begin{array}{c|c}
A_{i} & B_{i} \\
\hline C_{i} & D_{i}
\end{array}\right], \text { for } i=\Delta, d, f
$$

the corresponding standard model, takes the following form:

$$
\bar{G}=\left[\begin{array}{c|cccc}
\bar{A} & \bar{B}_{1} & \bar{B}_{2} & \bar{B}_{3} & \bar{B}_{4} \\
\hline \bar{C}_{1} & \bar{D}_{11} & \bar{D}_{12} & \bar{D}_{13} & \bar{D}_{14} \\
\bar{C}_{2} & \bar{D}_{21} & \bar{D}_{22} & \bar{D}_{23} & \bar{D}_{24} \\
\bar{C}_{3} & \bar{D}_{31} & \bar{D}_{32} & \bar{D}_{33} & \bar{D}_{34} \\
\bar{C}_{4} & \bar{D}_{41} & \bar{D}_{42} & \bar{D}_{43} & \bar{D}_{44}
\end{array}\right]
$$

where the inputs are $w_{1}, w_{2}, w_{3}$ and $u$, the outputs are $z_{1}, z_{2}, z_{3}$ and $y$. The involved matrices are as follows:

$$
\begin{aligned}
& \bar{A}=\left[\begin{array}{cccc}
A & B_{1} C_{\Delta} & B_{2} C_{d} & B_{3} C_{f} \\
0 & A_{\Delta} & 0 & 0 \\
0 & 0 & A_{d} & 0 \\
0 & 0 & 0 & A_{f}
\end{array}\right] \\
& {\left[\begin{array}{llll}
\bar{B}_{1} & \bar{B}_{2} & \bar{B}_{3} & \bar{B}_{4}
\end{array}\right]=} \\
& {\left[\begin{array}{cccc}
B_{1} D_{\Delta} & B_{2} D_{d} & B_{3} D_{f} & 0 \\
B_{\Delta} & 0 & 0 & 0 \\
0 & B_{d} & 0 & 0 \\
0 & 0 & B_{f} & 0
\end{array}\right]}
\end{aligned}
$$




$$
\begin{gathered}
\bar{C}_{1}=\left[\begin{array}{llll}
C_{1} & D_{11} C_{\Delta} & D_{12} D_{d} & D_{13} D_{f}
\end{array}\right] \\
\bar{D}_{1}=\left[\begin{array}{llll}
D_{11} D_{\Delta} & D_{12} D_{d} & D_{13} D_{f} & 0
\end{array}\right] \\
\bar{C}_{2}=\left[\begin{array}{c}
0 \\
0 \\
0 \\
-C_{f}
\end{array}\right], \bar{D}_{2}=\left[\begin{array}{llll}
0 & 0 & -D_{f} & I
\end{array}\right] \\
\bar{C}_{3}=\bar{C}_{2}, \bar{D}_{3}=\bar{D}_{2} . \\
\ddot{C}_{4}=\left[\begin{array}{llll}
C_{2} & D_{21} C_{\Delta} & D_{22} D_{d} & D_{23} D_{f}
\end{array}\right] \\
\bar{D}_{4}=\left[\begin{array}{llll}
D_{21} D_{\Delta} & D_{22} D_{d} & D_{23} D_{f} & 0
\end{array}\right]
\end{gathered}
$$

Note that $z_{3}$ is just a copy of $z_{2}$, thus making the system square (as required by some optimization procedures).

So far we have not been specific concerning the method of optimization to be employed for the standard problem (9).

Ideally speaking, the choice of method for optimization should be governed by the available data for the specific physical plant. E.g. if the disturbance model has been identified using bounded power amplification an $\mathcal{H}_{\infty}$ setup should be used, and if was obtained using time domain methods $\mathcal{L}_{1}$ methods would be the right choice.

However, very few methods are actually available that would effectively compute a useful filter for our problem. The reason is mainly that most robust methods are based on a robust stability/nominal performance principle. This is not appropriate for the problem we are considering, which is indeed an intricate one, because we are considering so many different effects at the same time (dynamical uncertainty, disturbances, noises, and faults). The only way to distinguish dynamical uncertainty from the other effects is to pose the problem as a robust performance problem. Robust stability/nominal performance methods would threat $w_{1}$ simply as an exogenous signal just as $w_{2}$ and $w_{3}$, and this approach would work only in very simple cases. Moreover, the disturbance model corresponding to (9) is based on structured uncertainty, which the method should also be able to handle.

The current state of art mainly offers two methods for design of robust performance in the face of structured uncertainty. These are $\mathcal{L}_{1}$ and $\mu$ optimization methods. In our opinion, these methods would be the best choices for small and medium size systems.

The following result describes the resulting behavior of a filter $F(s)$ designed by $\mu$ synthesis.

With some lack of stringence we will call the essential supremum of $\mu$ over all frequencies the $\mu$ norm. By the $\mathcal{L}_{1}$ norm we shall mean the operator norm of an operator mapping $\mathcal{L}_{\infty}$ signals (amplitude bounded) into $\mathcal{L}_{\infty}$ signals.
Theorem 1 Let $F(s)$ be a given transfer function. The following two are equivalent:

1. Applying the feedback $u=F(s) y$ for the system (9) stabilizes that system and makes the $\mu$ norm (the $\mathcal{L}_{1}$ norm) smaller than one with respect to the structure:

$$
\bar{\Delta}=\left[\begin{array}{ccc}
\Delta_{w} & 0 & 0 \\
0 & \Delta_{d} & 0 \\
0 & 0 & \Delta_{f}
\end{array}\right]
$$

2. Applying the filter $F(s)$ to the output of the original system (8) generates a signal $u$ with the property that the error signal $u-f$ has power bounded by 1 , provided ${ }^{*}$

(a) the $\mathcal{H}_{\infty}$ norm of the uncertainty weighted by the inverse of $W_{\Delta}$ is bounded by 1

(b) the power of $d$ weighted by the inverse of $W_{d}$ is bounded by 1

(c) the power of $f$ weighted by the inverse of $W_{f}$ is bounded by 1

A similar result holds for $\mathcal{L}_{1}$ optimization, simply by replacing the norms in the above theorem (and formulating it in time domain rather than frequency domain).

The multi objective method mentioned above will provide necessary conditions only for robust performance, but the interpretation will be the same as for the $\mu$ formulation.

The design algorithm we are putting forward is based on controller optimization techniques, although we are using these techniques to design a filter rather than a controller.

Algorithm 1 Indata: system matrices as in (9). Outdata: Filter $F(s)$.

1. Formulate weightings such that the faults would be isolated by the power signal interpretations in Theorem 1 (or by the corresponding time domain properties for $\mathcal{L}_{1}$ optimization)

2. Compute the matrices in order to set up the standard problem (9)

3. Apply $\mu$ synthesis (or $\mathcal{L}_{1}$ synthesis) to the problem (9)

4. The resulting controller $F(s)$ is the robust FDI filter for the system (8)

*The formulation of the last three conditions is not entirely stringent, since the inverses need not be neither stable nor proper. This slightly sloppy formulation is chosen to provide the intuition for the power signal interpretation, which would be relevant to an FDI application. 


\section{Conclusion}

The failure detection and isolation in presence of modeling errors and disturbance problem has been considered in this paper by using two different approaches.

The first approach applied to the FDI problem is based on using observers together with a prefilter. This design approach has been formulated as a robust standard design problem. In previous approaches in the literature, the observer and the prefilter was designed separately, which does not guarantee optimality. If instead we use the robust standard formulation, the design of observer and prefilter is done in one step. However, for making an optimal filter for the FDI problem, we need only the prefilter. So, as the result of this approach, the observer based approach is equivalent with a standard filter design in the optimal case, but it offers additional information which might be desirable in itself.

The other approach is based on a direct formulation of the FDI design problem, and offers a completely optimization based design of an unstructured filter, which need not be observer based in any intuitive way.

Both approaches offer implementation under several optimization schemes which might be given by the application. It is argued that from current stateof-the-art methodologies, $\mu$ or $\mathcal{L}_{1}$ based methods would yield the best results for FDI applications, such as the one addressed in this paper.

Depending on how large the design problem is (number of states, number of inputs and outputs etc.), it might be more convenient to use a simpler multi objective design approach based on sensitivity optimization, rather than $\mathcal{H}_{\infty}, \mu$, or $\mathcal{L}_{1}$ methods for computational reasons.

\section{References}

[1] H. Ajbar and J.C. Kantor. An $l_{\infty}$ approach to robust control and fault detection. In Proceedings of the American Control Conference, pages 3197-3201, San Francisco, CA, USA, 1993.

[2] M. Blanke, S.A. Bøgh, R.B. Jørgensen, and R.J. Patton. Fault detection for a diesel engine actuator - A benchmark for FDI. Control Eng. Practice, 3:1731-1740, 1995.

[3] E.A.J. Bokor and L. Keviczky. An $\mathcal{H}_{\infty}$ filtering approach to robust detection of failures in $\mathrm{dy}$ namical systems. In Proceedings of the 33rd Conference on Decision and Control, pages 30373039, Lake Buena Vista, FL, USA, 1994.
[4] P.M. Frank. Fault diagnosis in dynamic systems using analytic and knowledge-based redundancy - A survey and some new results. AUTOMATICA, 26:459-474, 1990.

[5] P.M. Frank and X. Ding. Frequency domain approach to optimally robust residual generation and evaluation for model-based fault diagnosis. AUTOMATICA, 30:789-804, 1994.

[6] E.A. Garcia, B. Köppen-Seliger, and P.M. Frank. A frequency domain approach to residual generation for the industrial actuator benchmark. Control Eng. Practice, 3:1747-1750, 1995.

[7] R.W. Grainger, J. Holst, A.J. Isaksson, and B.M. Ninness. A parametric statistical approach to FDI for the industrial actuator benchmark. Control Eng. Practice, 3:1757-1762, 1995.

[8] R.B. Jørgensen, R.J. Patton, and J. Chen. An eigenstructure assignment approach to FDI for the industrial actuator benchmark test. Control Eng. Practice, 3:1751-1756, 1995.

[9] R.S. Mangoubi, B.D. Appleby, G.C. Verghese, and W.E. VanderVelde. A robust failure detection and isolation algorithm. In Proceedings of the 34th Conference on Decision and Control, pages 2377-2382, New Orleans, LA, USA, 1995.

[10] R. Patton, P. Frank, and R. Clark. Fault diagnosis in dynamic systems - Theory and application. Prentice Hall, 1989.

[11] R.J. Patton and J. Chen. Robust fault detection using eigenstructure assignment: A tutorial consideration and some new results. In Proceedings of the 30th Conference on Decision and Control, pages 2242-2247, Brighton, England, 1991.

[12] Z. Qiu and J. Gertler. Robust FDI systems and $\mathcal{H}_{\infty}$ optimization. In Proceedings of the 32nd Conference on Decision and Control, pages 1710-1715, San Antonio, Texas, USA, 1993.

[13] Y.Y. Wang and N.E. Wu. An approach to configuration of robust control systems for robust failure detection. In Proceedings of the 32nd Conference on Decision and Control, pages 1704-1709, San Antonio, Texas, USA, 1993. 